\title{
Surveillance for Hepatocellular Carcinoma: Long Way to Achieve Effectiveness
}

\author{
Hashem B. El-Serag
}

Received: 19 August 2012 / Accepted: 22 August 2012/Published online: 6 October 2012

(C) Springer Science+Business Media, LLC (Outside the USA) 2012

Surveillance for hepatocellular carcinoma (HCC) in high risk groups including those with cirrhosis as well as with chronic HBV infection is recommended by several practice guidelines including those by the Asian Pacific Association for the Study of Liver (APASL) [1], the American Association for the Study of Liver Disease (AASLD) and the European Association for Study of The Liver (EASL). The evidence supporting the surveillance recommendations in these guidelines is based on evidence of intermediate strength, although arguably the evidence is strongest for HCC surveillance in individuals infected with HBV in Southeast Asia. For example, two randomized controlled trials were conducted in China among individuals with chronic HBV infection with and without cirrhosis. In one placebo-controlled randomized study of nearly 19,000 HBV-infected patients, it was shown that HCC surveillance with both abdominal ultrasound and serum AFP repeated at 6-month intervals resulted in a $37 \%$ reduction in HCCrelated mortality [2]. However, the other randomized controlled trial of 5,581 HBV-infected patients reported that serum AFP repeated at 6-month intervals did not result in a significant reduction in overall mortality [3]. The latter study was smaller and had detected HCC less frequently than the first study and therefore could have suffered from a lack of adequate statistical power. In addition to these two randomized trials, a population-based surveillance program using serum AFP every 6-months among HBVinfected patients in Alaska reported that, during a 16-year

\section{H. B. El-Serag ( $\square)$}

Dan L Duncan Professor of Medicine, Michael E DeBakey

VA Medical Center, Houston Center for Quality of Care

and Utilization Studies, Baylor College of Medicine,

Houston, TX, USA

e-mail: hasheme@bcm.edu follow-up period, patients with HCC who received surveillance had significantly longer 5-year survival compared with historical controls (42 vs. $0 \%$, respectively) [4]. Last in the evidence hierarchy, several nonrandomized trials and observational cohort and case-control studies have reported that patients who undergo HCC surveillance are diagnosed at an earlier stage of $\mathrm{HCC}$, are more likely to receive potentially curative therapy, and have a significant reduction in cancer-specific mortality compared with patients detected with symptomatic HCC [5, 6].

The "efficacy" of HCC surveillance reflects the degree to which surveillance produces the expected result under controlled conditions chosen to maximize the likelihood of observing an effect if it exists. By contrast, "effectiveness" addresses the extent to which HCC surveillance is beneficial when deployed in medical practice settings [7]. Effectiveness takes into account external factors such as individual patient characteristics, health system features, and societal influences. For HCC surveillance to be effective, several steps need to be enacted. These include (1) identification of high risk patients who are appropriate for surveillance (HBV and HCV with advanced fibrosis/ cirrhosis), (2) availability and accessibility of HCC surveillance to patients at risk in appropriate health care settings, (3) recommendation of the intervention by health care providers, (4) acceptance of HCC surveillance by patients, (5) adherence to surveillance, recall and follow-up at the recommended intervals, the benefits of therapy, (6) availability of appropriate diagnostic test follow-up for abnormal surveillance, and (7) availability of efficacious curative or palliative treatment once HCC is diagnosed. Given the multiplicity of components involved with determining HCC surveillance effectiveness, one could assess the interactions between these factors to arrive with an estimate of how one particular intervention would 
perform in everyday practice. Under a hypothetical scenario, the overall effectiveness drops with small decrements in the rates of $\mathrm{HCV}$ or HBV diagnosis (e.g., only $70 \%$ get tested), access to surveillance ( $80 \%)$, recommendation for surveillance $(80 \%)$, acceptance by patients $(80 \%)$, adherence to recommended intervals ( $80 \%$ ), proper followup $(80 \%)$ and timely availability of diagnostic and therapeutic options $(80 \%)$. Assuming that these are independent probabilities and thus multiplying them by each other and by the estimated efficacy of HCC surveillance in clinical trials (i.e., $35 \%$ ), the resulting effectiveness could be as low as $6 \%(0.35 \times 0.7 \times 0.8 \times 0.8 \times 0.8 \times 0.8 \times 0.8 \times 0.8)$.

The study by Park et al. [8] reported the results of a survey in the Republic of Korea, an area endemic for HBV (seroprevalence of the HBV surface antigen was $4.1 \%$ ). Among the $436 \mathrm{HBV}$-infected persons, approximately $27 \%$ were up to date with HCC screening, but more than half $(52.9 \%)$ had never been screened. Self-reported awareness of HBV infection was significantly associated with current $\mathrm{HCC}$ screening (odds ratio $=2.82 ; 95 \% \mathrm{CI}$ 1.64-4.84). This finding is in agreement with other studies from the United States indicating that the extent of utilizing HCC surveillance in clinical practice is low. In a study of $13,002 \mathrm{HCV}$-infected US veterans diagnosed with cirrhosis during 1998-2005, only $12 \%$ received annual surveillance in the 3 years following their cirrhosis diagnosis, and less than $50 \%$ received a surveillance test in the first year following cirrhosis diagnosis [9]. Several retrospective US studies among patients with newly diagnosed HCC also found very low rates of surveillance prior to their HCC diagnosis $[10,11]$.

The study by Park et al. and other studies highlight that the most basic step in the cascade between efficacy and effectiveness, namely screening for HBV (and HCV in US studies) and informing patients of their status (positive or negative), is shockingly defective. In addition, these studies show that, even among high risk groups, there is low utilization of HCC surveillance. The reasons for these additional drop-offs are unclear and are currently the subject of examination from multiple groups, but likely reflect a combination of cognitive (knowledge of surveillance and diagnosis methods or therapeutic options) as well as logistical factors, such as the need for repeated testing over relatively short periods of time, somewhat complicated diagnostic evaluation for $\mathrm{HCC}$, and the limited availability of potentially curative options such as liver transplant centers to refer patients who are diagnosed with HCC. These obstacles need to be identified and tackled for the successful implementation of any effective HCC surveillance program.

\section{References}

1. Omata M, Lesmana LA, Tateishi R, et al. Asian Pacific Association for the Study of the Liver consensus recommendations on hepatocellular carcinoma. Hep Intl. 2010;4:439-474.

2. Zhang BH, Yang BH, Tang ZY. Randomized controlled trial of screening for hepatocellular carcinoma. J Cancer Res Clin Oncol. 2004;130:417-422.

3. Chen JG, Parkin DM, Chen QG, et al. Screening for liver cancer: results of a randomised controlled trial in Qidong, China. J Med Screen. 2003;10:204-209.

4. McMahon BJ, Bulkow L, Harpster A, et al. Screening for hepatocellular carcinoma in Alaska natives infected with chronic hepatitis B: a 16-year population-based study. Hepatology. 2000;32:842-846.

5. Trevisani F, De NS, Rapaccini G, et al. Semiannual and annual surveillance of cirrhotic patients for hepatocellular carcinoma: effects on cancer stage and patient survival (Italian experience). Am J Gastroenterol. 2002;97:734-744.

6. Yu EW, Chie WC, Chen TH. Does screening or surveillance for primary hepatocellular carcinoma with ultrasonography improve the prognosis of patients? Cancer J. 2004;10:317-325.

7. El-Serag HB, Talwalkar J, Kim WR. Efficacy, effectiveness, and comparative effectiveness in liver disease. Hepatology. 2010;52:403-407.

8. Park SH, Heo NY, Park JH, et al. Hepatocellular carcinoma screening in a hepatitis B virus-infected Korean population. Dig Dis Sci. (Epub ahead of print). doi:10.1007/s10620-012-2281-6.

9. Davila JA, Henderson L, Kramer JR, et al. Utilization of surveillance for hepatocellular carcinoma among hepatitis $\mathrm{C}$ virusinfected veterans in the United States. Ann Intern Med. 2011;154:85-93.

10. Davila JA, Morgan RO, Richardson PA, Du XL, McGlynn KA, El-Serag HB. Use of surveillance for hepatocellular carcinoma among patients with cirrhosis in the United States. Hepatology. 2010;52:132-141.

11. Davila JA, Weston A, Smalley W, El Serag HB. Utilization of screening for hepatocellular carcinoma in the United States. J Clin Gastroenterol. 2007;41:777-782. 